\title{
Impacto da Introdução de Mídia Eletrônica num Curso de Patologia Geral
}

\author{
Impact of the Introduction of Electronic \\ Media in a General Pathology Course
}

Flávia Branco Cerqueira Serra Neves ${ }^{I}$

Camila Silva Bôaventura ${ }^{I}$

Almir Galvão Vieira Bitencourt ${ }^{\text {II }}$

Daniel Abensur Athanazio ${ }^{I I}$

Mitermayer Galvão dos Reis ${ }^{I I I}$

PALAVRAS-CHAVE:

- Patologia;

- Educação Médica;

- Modelos Educacionais.

\section{KEY WORDS:}

- Pathology;

- Medical Education;

- Educational Models.

Recebido em: 15/03/2007

Reencaminhado em: 17/07/2007

Aprovado em: 16/01/2008

REVISTA BRASILEIRA DE EDUCAÇÃO MÉDICA
${ }^{1}$ Escola Bahiana de Medicina e Saúde Pública, Bahia, Brasil.

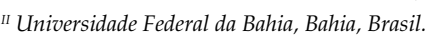

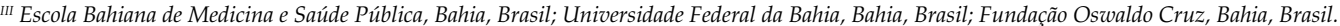




\section{INTRODUÇÃO}

A Patologia representa um componente-chave da formação médica, servindo tanto como uma ponte entre as ciências básicas e a prática clínica, quanto como referência fundamental para a pesquisa básica. Uma característica fundamental da Patologia compartilhada por poucas outras disciplinas é a necessidade de reconhecer aspectos morfológicos tanto macro quanto microscópicos. Assim, tradicionalmente, a disciplina é apresentada aos estudantes de Medicina a partir de uma combinação de exposições teóricas e práticas com peças macroscópicas e lâminas histológicas.

A Patologia abrange o estudo dos mecanismos de doença, bem como o efeito desta nos órgãos e tecidos (Anatomia Patológica). Um problema aparentemente universal nas escolas médicas é a percepção de que o aprendizado da Anatomia Patológica é difícil ${ }^{1}$. Recentemente, este problema vem se agravando em decorrência do avanço da medicina de alta complexidade, que cria um contexto em que todo conhecimento que não está claro e diretamente relacionado à prática profissional futura não desperta o interesse dos estudantes².

A incorporação dos computadores, da internet e CD-ROM ao ensino da graduação médica vem sendo recentemente estimulada ${ }^{3}$. Mas a falta de integração com outras formas de ensino/aprendizado tradicionalmente utilizadas e a resistência cultural dos alunos e educadores são fatores que podem prejudicar a introdução de novas tecnologias de ensino ${ }^{4,5}$.

No Brasil, foram implementadas muitas iniciativas de investimento em educação assistida por computador, mas são escassas a publicação de resultados e a discussão sobre a experiência internacional ${ }^{6}$. Um exemplo brasileiro é a universidade de Londrina, no Paraná, onde um atlas do sistema osteoarticular e muscular em CD-ROM dinamizou as aulas ${ }^{7}$. Experiências no âmbito da Patologia com uso de material suplementar, com base em educação assistida por computador, se iniciaram em Michigan em $1986^{\circ}$, e o ensino via web faz parte hoje da rotina de outras universidades americanas com boa avaliação pelos estudantes, como acontece na Filadélfia9.

O objetivo deste trabalho é avaliar a aceitação por parte dos alunos e o impacto de modificações implementadas recentemente num curso de Patologia Geral, com vistas a incentivar a autoinstrução dos alunos por meio de recursos eletrônicos, e fazer uma revisão das concepções atuais sobre o ensino da Patologia.

\section{METODOLOGIA}

\section{Desenho do estudo e população}

Foi realizado um estudo descritivo, de corte transversal, em que foram avaliados estudantes da Escola Bahiana de Medicina e Saúde Pública (EBMSP), ao final da disciplina de Pa- tologia Geral, ministrada no quarto semestre do curso. Foram incluídos os estudantes de três turmas consecutivas após a implementação de mudanças no curso.

\section{Contexto}

A disciplina de Patologia é ensinada na EBMSP em dois semestres. O primeiro abrange a Patologia Geral, disciplina do segundo ano, e o segundo aborda a Patologia dos Sistemas, disciplina do terceiro ano, já integrada à Semiologia Médica, com discussão conjunta de casos em sessões anatomoclínicas. Até 2003, a estrutura do curso de Patologia Geral obedecia à tradicional divisão de aulas expositivas, estudos dirigidos com pequeno grupo de alunos sobre temas de cada módulo e aulas práticas de macro e microscopia ${ }^{3}$. A partir de então, foram implementadas modificações na disciplina com o objetivo de estimular a participação ativa dos alunos em seu aprendizado.

\section{Intervenção}

Como recurso adicional de estudo e com o objetivo de propiciar ao corpo discente um melhor aprendizado do conteúdo das aulas práticas e teóricas, foi distribuído a todos os estudantes um CD-ROM criado pelos professores da disciplina. Este instrumento continha todos os arquivos das aulas teóricas, um atlas de Patologia Geral com lâminas do curso e outros exemplos estudados nos módulos, mas não apresentados em aulas práticas, e uma seleção de publicações científicas que complementam os livros-texto, para aprofundamento de temas específicos de interesse dos alunos ${ }^{10}$.

Outra atividade paralela foi o maior investimento na monitoria. Foram oferecidas mais vagas para monitoria, e os professores destinaram mais tempo a reuniões prévias e à revisão de lâminas. As sessões semanais ou quinzenais de monitoria sempre incluíam a revisão da histologia das próximas aulas práticas, bem como a apresentação, por um monitor, de um trabalho científico atual e relevante dentro do módulo específico de Patologia Geral.

A avaliação do desempenho do aluno passou a ser realizada por quatro provas teóricas, uma prova prática no fim do semestre e uma nota baseada no desempenho nos estudos dirigidos semanais. A prova prática consistia na análise de duas lâminas histológicas acompanhadas de questões sobre como identificar o tecido normal e como classificar o processo patológico presente. As lâminas usadas nas avaliações incluíam os mesmos tecidos e processos patológicos vistos durante o curso prático, em diferentes combinações, mas não se tratava do mesmo material visto pelos alunos durante o semestre letivo. 


\section{Coleta dos dados}

Os concluintes de cada semestre foram solicitados a preencher um questionário informando seu desempenho na avaliação prática e como eles próprios avaliavam o curso (Anexo). As turmas referidas como 1, 2 e 3 são as que cursaram Patologia Geral nos semestres de 2005-1, 2005-2 e 2006-1. As respostas foram acompanhadas por assinatura de Termo de Consentimento Livre e Esclarecido para uso das informações. O projeto foi aprovado pelo Comitê de Ética Médica da EBMSP, com número de protocolo 13/2006.

\section{Procedimentos de análise}

$\mathrm{Na}$ análise dos dados, foi utilizado o software Statistical Packcage for Social Science (SPSS) na versão 9.0. Foram utilizados parâmetros da estatística descritiva, adotando-se as medidas usuais de tendência central e de dispersão, e cálculos de freqüências simples e relativas. Para a correlação entre as variáveis, foram utilizados os testes Qui-Quadrado ou exato de Fisher para as freqüências das variáveis categóricas, teste $\mathrm{T}$ de Student para as variáveis contínuas com distribuição normal, e Mann-Whitney para as variáveis contínuas sem distribuição normal. O nível de significância adotado foi de 5\%.

\section{RESULTADOS}

Com os resultados das três turmas, foram avaliados 288 estudantes, sendo $53,0 \%$ do gênero feminino, com idade média de 20,98 $\pm 2,03$ anos. A maioria dos estudantes $(94,4 \%$ ) considera importante a presença do monitor nas aulas práticas e $68,2 \%$ avaliaram a participação dos monitores como boa ou muito boa. A principal fonte de estudo para a prova prática utilizada pelos estudantes foi o CD distribuído pela disciplina em 97,2\% dos casos, seguido do livro-texto de Patologia $(60,1 \%)$, atlas de Histologia (34,0\%) e atlas de Patologia (9,7\%). A importância média dada ao CD foi de $8,45 \pm 1,79$, numa escala de 0 a 10 (primeira turma: 7,8 $\pm 2,2$; segunda turma: 8,9 $\pm 1,2$; terceira turma: $8,7 \pm 1,6)$. Grande parte dos estudantes $(93,1 \%)$ referiu ter computador em casa, $91,3 \%$ têm acesso à internet em casa e $82,2 \%$ relataram utilizar a internet para complementar o aprendizado da disciplina.

Quanto à avaliação do curso, 95,8\% dos alunos acharam que o curso prático de Patologia melhorou sua capacidade de reconhecer tecidos ou órgãos ao microscópio. A prova prática, como método de avaliação de aprendizagem do conteúdo, foi avaliada como boa ou muito boa por $37,6 \%$ dos estudantes, regular por $34,8 \%$ e ruim ou muito ruim por $27,5 \%$. Após o curso prático de Patologia Geral, 42,4\% dos estudantes referiram ter conseguido identificar todos os processos gerais, como necrose, inflamação e neoplasia, 56,6\%
TABELA 1

Resultados da avaliação das modificações do curso de Patologia Geral das turmas avaliadas

\begin{tabular}{|c|c|c|c|}
\hline & $\begin{array}{c}1^{\text {a }} \text { turma } \\
(\mathrm{n}=96)\end{array}$ & $\begin{array}{l}2^{a} \text { turma } \\
(n=100)\end{array}$ & $\begin{array}{c}3^{\text {a }} \text { turma } \\
(\mathrm{n}=92)\end{array}$ \\
\hline $\begin{array}{l}\text { Considerou importante a presença } \\
\text { do monitor na aula }\end{array}$ & $91,5 \%$ & $97,0 \%$ & $94,6 \%$ \\
\hline $\begin{array}{l}\text { Avaliou a participação dos } \\
\text { monitores como boa ou muito boa }\end{array}$ & $60,0 \%$ & $75,0 \%$ & $69,3 \%$ \\
\hline Estudou pelo CD & $95,8 \%$ & $98,0 \%$ & $97,8 \%$ \\
\hline $\begin{array}{l}\text { Utilizou internet para } \\
\text { complementar estudo }\end{array}$ & $71,9 \%$ & $84,8 \%$ & $90,2 \%$ \\
\hline $\begin{array}{l}\text { Houve melhora da capacidade } \\
\text { de reconhecer órgãos e tecidos no } \\
\text { microscópio }\end{array}$ & $90,6 \%$ & $99,0 \%$ & $97,8 \%$ \\
\hline $\begin{array}{l}\text { Conseguiu identificar durante } \\
\text { o curso todos os processos } \\
\text { patológicos básicos, como necrose, } \\
\text { inflamação e neoplasia }\end{array}$ & $39,6 \%$ & $49,0 \%$ & $38,1 \%$ \\
\hline $\begin{array}{l}\text { Avaliou a forma de avaliação como } \\
\text { boa ou muito boa }\end{array}$ & $21,0 \%$ & $53,0 \%$ & $38,0 \%$ \\
\hline Notas inferiores a 6,0 & $43,7 \%$ & $39,0 \%$ & $42,4 \%$ \\
\hline
\end{tabular}

conseguiram identificar apenas alguns desses processos e $1,0 \%$ nunca conseguiu identificá-los. A maioria dos alunos (96\%) acha que a visualização desses processos no curso de Patologia Geral auxilia no estudo da Patologia dos Sistemas. Quando questionados sobre a prática médica futura, 62,5\% dos estudantes referiram que entenderão facilmente um laudo anatomopatológico e 37,5\% terão que estudar para entender os termos referentes aos processos gerais de patologia e suas subclassificações.

Os alunos da primeira turma avaliaram pior as modificações implementadas no curso em relação aos estudantes das duas turmas posteriores (Tabela 1). A utilização de mídia eletrônica foi bem avaliada por estudantes de ambos os sexos, que tinham ou não computador e acesso à internet em casa. Os alunos que tiveram notas acima de 6,0 na avaliação prática avaliaram melhor a importância do CD (média de 8,70 x 8,14 numa escala de 1 a $10, P=0,009$ ) e a forma de avaliação (média de 3,39 x 2,58 numa escala de 1 a 5, $P<0,001$ ), quando comparados aos que apresentaram rendimento inferior. Por outro lado, os que estudaram pelo CD (97,2\% do total) apresentaram menor freqüência de notas baixas - consideradas inferiores a $6,0(39,9 \% \times 87,5 \%, P=0,010)$ - e identificaram os processos gerais com maior freqüência $(99,3 \% \mathrm{x}$ $87,5 \%, P=0,012)$ em relação aos que não utilizaram este método (Gráfico 1). 
GRÁFICO 1

Freqüência de notas baixas $(<6,0)$ dos alunos que estudaram ou não pelo CD-ROM.

$\square$ Não estudaram pelo CD-ROM
$\square$ Estudaram pelo CD-ROM

\section{DISCUSSÃO}

Os estudantes avaliaram positivamente as modificações implementadas no curso, atribuindo elevada importância à presença dos monitores nas aulas práticas e ao CD-ROM distribuído. Além disso, o curso cumpriu o objetivo de melhorar a capacidade de reconhecer órgãos e tecidos no microscópio, bem como de identificar e entender os principais tipos de processos patológicos, sendo esta avaliação melhor a partir da segunda turma. Houve boa aceitação do CD-ROM distribuído pela disciplina e melhor rendimento na avaliação quando o CD foi empregado como instrumento de estudo. No entanto, outros fatores, além da utilização do $\mathrm{CD}$, podem ter contribuído para o rendimento no aprendizado e a valoração do sistema de ensino e aprendizado, como: eficácia da assistência do monitor, interesse na matéria, assiduidade às práticas, tempo de dedicaçao ao estudo e base geral de conhecimentos dos participantes. Os alunos que apresentaram baixo rendimento avaliaram pior tanto o CD-ROM como o método de avaliação do curso.

Duas limitações neste contexto são a falta de interesse pelas aulas práticas e atividades de discussão com professores num curso com avaliação predominantemente teórica e uma forma objetiva de avaliar o desempenho dos alunos no curso prático. Estudos apontam uma tendência de concentração de acessos às tecnologias eletrônicas quando se aproximam as avaliações da disciplina. Estes achados justificam a dificuldade de incorporar uma nova cultura de aprendizagem, já que os estudantes apresentam um pragmatismo acentuado quanto à obtenção de notas nas provas como objetivo principal na disciplina ${ }^{5,11}$.

A internet oferece inúmeras fontes que suplementam um programa teórico básico de Patologia Geral, com imagens de macro e microscopia de qualidade, a exemplo das citadas na Quadro 1. No entanto, o excesso de informação disponível pode confundir mais do que facilitar o aprendizado de um aluno não devidamente instruído. Além do mais, uma série de endereços eletrônicos de acurácia duvidosa, com informações não referenciadas ou superficiais, é um problema para estudantes de qualquer área. Um sistema de classificação de sites dedicados à Patologia foi, inclusive, proposto com base em critérios como acurácia das informações, facilidade de navegação, relevância, atualização e profundidade ${ }^{12}$. Por outro lado, assim como outros autores ${ }^{8}$, testemunhamos a vantagem de usar mídia eletrônica própria, pois o material pode ser organizado de acordo com os módulos, casos, situações e lâminas histológicas observadas durante o curso. Dessa forma, uma vez sedimentado o conhecimento básico oferecido em sala de aula, a internet fornece um imenso conteúdo adicional para pesquisa, preparação de trabalhos e auto-instrução.

Optamos pela distribuição de CD-ROM ao invés da disponibilização pela internet especialmente pela questão técnica, visto que uma limitação importante para o sucesso de experiências semelhantes é a velocidade de acesso ${ }^{13}$. Como muitos arquivos são grandes e nem todos os alunos têm disponibilidade de conexões rápidas, a opção do CD-ROM tornou todo o material rapidamente acessível para todos que possuem computador ou estudam no laboratório de informática da escola.

No CD-ROM, tentamos sempre apresentar imagens do tecido normal, comparando-o com o tecido patológico em graus de magnificações crescentes de áreas características. No entanto, nem uma bem elaborada série de imagens e legendas substitui o conhecimento prático em que o aluno seja capaz de, no microscópio, reconhecer o normal e achar a patologia. Marchevsky e colaboradores reconhecem que uma limitação importante ao substituir práticas de módulos de Patologia Sistêmica por mídia eletrônica é a falta de contato com o mi- 


\section{QUADRO 1}

Sites na internet com material de Patologia recomendados

\begin{tabular}{|c|c|}
\hline Fonte & Endereço eletrônico e comentários \\
\hline Universidade de Illinois & $\begin{array}{l}\text { http://www.med.uiuc.edu/histo/small/atlas/slides.htm } \\
\text { Atlas interativo de Histologia }\end{array}$ \\
\hline Universidade Federal da Bahia & $\begin{array}{l}\text { http://www.medicina.ufba.br/patologia_i/welcome.htm } \\
\text { Atlas de Patologia Geral em português }\end{array}$ \\
\hline Universidade de Campinas & $\begin{array}{l}\text { http://www.fcm.unicamp.br/departamentos/anatomia/ } \\
\text { Extensa coleção de imagens de macro e microscopia referentes a Patologia Geral e Sistêmica em português }\end{array}$ \\
\hline Universidade da Flórida & $\begin{array}{l}\text { http:/ / www-medlib.med.utah.edu/WebPath/webpath.html } \\
\text { Atlas de Patologia Geral e Sistêmica, com destaque para excelentes imagens de macroscopia de peças } \\
\text { cirúrgicas a fresco }\end{array}$ \\
\hline Pathology Outlines & $\begin{array}{l}\text { http:/ / www.pathologyoutlines.com/ } \\
\text { Lista de temas de Patologia com conexões para outros sites de acesso livre }\end{array}$ \\
\hline Universidade de Pittsburg & $\begin{array}{l}\text { http:/ / www.path.upmc.edu/cases.html } \\
\text { Extensa coleção de casos com correlação de aspectos clínicos e morfológicos }\end{array}$ \\
\hline
\end{tabular}

croscópio. Isto restringirá o conhecimento do aluno de como os tecidos são analisados, quais as limitações desta análise ou como o normal e o patológico interagem num mesmo microambiente ${ }^{14}$. O reconhecimento de que as apresentações morfológicas podem ser variáveis, além da existência de artefatos e outras patologias, pode, inclusive, confundir uma interpretação histológica. Recentemente, a tecnologia evoluiu para criar o "microscópio virtual", ou seja, um corte histológico pode ser fotografado por inteiro e apresentado com uma imagem interativa, onde podemos clicar em qualquer ponto e aumentá-lo, como num microscópio convencional ${ }^{15,16}$.

Embora a perda de contato com o microscópio e com a habilidade de manuseá-lo permaneça uma questão importante, a mídia eletrônica ganha muito em interatividade. Com experiência de dez anos de aulas práticas híbridas, com microscopia convencional e discussão de "lâminas virtuais", os autores reforçam a idéia de que a aplicação de novas tecnologias apenas dinamiza as sessões e é amplamente bem aceita pelos alunos. Mas esse modelo não abole o contato dos alunos com o microscópio convencional e não está inserido num contexto de redução de professores especialistas na área ou de redução do tempo de contato entre alunos e instrutores ${ }^{16}$.

Acreditamos que o emprego de novas ferramentas só seja útil numa hierarquia que estimule primeiramente a autoinstrução do aluno no conteúdo teórico, a discussão com os professores e a participação na aula prática com professores e monitores preparados. A partir daí, a mídia, integrada ao que foi contemplado no módulo didático, oferece ao aluno a ca- pacidade permanente de revisar o tema. Só então, a internet pode ser explorada por um indivíduo preparado para uma fonte de informações praticamente inesgotável.

\section{CONCLUSÕES}

As modificações implementadas na disciplina foram avaliadas positivamente pelos estudantes, contribuindo para um melhor desempenho destes nas avaliações e um melhor aproveitamento do curso. O CD-ROM oferece recursos que podem facilitar o processo ensino-aprendizagem, representando uma alternativa para o ensino integrado de Patologia Geral. No entanto, o aproveitamento das oportunidades oferecidas pelas tecnologias digitais depende fundamentalmente de mudanças no padrão pedagógico dos cursos, no sentido de estimular o alunado a assumir de fato a condição de sujeito ativo do processo ensino-aprendizagem.

\section{AGRADECIMENTOS}

Gostaríamos de agradecer a todos os monitores da disciplina de Patologia Geral pelo interesse e participação no curso no período aqui descrito, bem como aos outros docentes de Patologia da EBMSP.

\section{REFERENCIAS}

1. Damjanov I. Teaching of pathology at more than one level. Hum pathol 2005;36(2): 135-138.

2. Nash JR, West KP, Foster CS. The teaching of anatomic pathology in England and Wales: a transatlantic view. Hum pathol. 2001;32(11):1154-1156. 
3. World Federation for Medical Education (WFME) guidelines for using computers in medical education. Med educ. 1998;32(2):205-208.

4. Greenhalgh T. Computer assisted learning in undergraduate medical education. BMJ. 2001;322(7277):40-44.

5. Coeli CM, Almeida LM, Rocha TCG, Camargo-Junior KR. Epidemio Online: um site de apoio ao processo ensinoaprendizagem de epidemiologia na graduação de Medicina. Rev bras educ med. 2004;28(3):259-263.

6. Adler MD, Johnson KB. Quantifying the literature of computer-aided instruction in medical education. Acad med. 2000;75(10):1025-1028.

7. Fornaziero CC, Gil CRR. Novas tecnologias aplicadas ao ensino da anatomia humana. Rev bras educ med. 2003;27(2):141-146.

8. Kumar K, Obielodan J. Long-term experience and evaluation of computer software for general pathology laboratory case studies. Pathol educ 2000;25(1):20-24.

9. Frisby AJ, Fenderson BA, Damjanov I, Braster CD, Murray RB. Course activities: using World Wide Web technology to supplement traditional pathology instruction. Pathol educ 1997;22(1):16-23.

10. Neves FBCS, Boâventura C, Guimarães A, Athanazio DA, Reis MG. Introdução de novos métodos de ensino na disciplina de Patologia Geral da EBMSP. Revista Digital de Educação Médica Permanente em Saúde 2004;1(Suppl.1):165-166.

11. Peixoto MAP, Mattos TM. Fontes de estudo na escola médica - recursos instrucionais utilizados por alunos de medicina da região sudeste do Brasil. Rev bras educ med 2000;26(1):28-34.
12. Talmon G, Abrahams NA. The Internet for pathologists: a simple schema for evaluating pathology-related Web sites and a catalog of sites useful for practicing pathologists. Arch Pathol lab med 2005;129(6):742-6.

13. McLay RN, Bowdish B, Clejan S, Crawford B. Building a better web page incorporating the results of student survey's to improve a medical school pathology site. Pathol educ 2004;27(1):39-47.

14. Marchevsky AM, Relan A, Baillie S. Self-instructional "virtual pathology" laboratories using web-based technology enhance medical school teaching of pathology. Hum pathol 2003;34(5):423-9.

15. Dick FR. Web-based virtual microscope laboratories. Pathol educ 2000;25(2):58-62.

16. Kumar RK, Velan GM, Korell SO, Kandara M, Dee FR, Wakefield D. Virtual microscopy for learning and assessment in pathology. J Pathol 2004;204(5):613-618

ANEXO: O questionário utilizado está disponível para consulta em http:/ / www.educacaomedica.org.br/Anexos

\section{CONFLITOS DE INTERESSE}

Declarou não haver.

\section{ENDEREÇO PARA CORRESPONDÊNCIA}

Flávia Branco Cerqueira Serra Neves

Av. Orlando Gomes, 382 - B-18

Condomínio Village Piatã - Piatã

CEP.: 41650-010 Salvador / BA

E-mail: flavinhaneves@superig.com.br 